\title{
The Effects of Variations in Jet Width on the Growth of Baroclinic Waves: Implications for Midwinter Pacific Storm Track Variability
}

\author{
NILI HARNIK \\ Lamont-Doherty Earth Observatory, Columbia University, Palisades, New York \\ EdMund K. M. Chang \\ ITPA/MSRC, State University of New York at Stony Brook, Stony Brook, New York
}

(Manuscript received 3 February 2003, in final form 1 July 2003)

\begin{abstract}
The effects of variations in jet width on the downstream growth of baroclinic waves are studied, using a simple quasigeostrophic model with a vertically varying basic state and variable channel width, as well as a simplified primitive equation model with a basic state that varies in latitude and height. This study is motivated by observations that in midwinter in the Pacific the storm track is weaker and the jet is narrower during years when the jet is strong.

The linear models are able to reproduce the observed decrease of spatial growth rate with shear, if the narrowing of the jet is accounted for by assuming it decreases the meridional wavelength of the perturbations, which hampers their growth. A common suggestion has been that perturbations are weaker when the jet is strong because they move faster out of the unstable storm track region. The authors find that one needs to take into account that the jet narrows when it strengthens; otherwise, the increase of growth rate is strong enough to counteract the effect of increased advection speed.

It is also found that, when the model basic state is Eady-like (small or zero meridional potential vorticity gradients in the troposphere), the short-wave cutoff for instability moves to large-scale waves as shear is increased, due to the accompanying increase in meridional wavenumber. This results in a transition from a regime where upper-level perturbations spin up a surface circulation very rapidly, and normal-mode growth ensues, to a regime where the initial perturbations take a very long time to excite growth. Since waves slow down when a surface perturbation develops, this can explain the observations that the storm track perturbations are more "upper level" during strong jet years and their group velocities increase faster than linearly with shear.
\end{abstract}

\section{Introduction and motivation}

In this paper we study the effects of jet width on the growth of baroclinic waves in a storm track setup, with the goal of understanding the observation that the midwinter storm track is stronger during years when the jet is weak. Nakamura (1992) was the first to show that the intensity of the storm track is positively correlated to jet strength for jet wind speeds below $45 \mathrm{~m} \mathrm{~s}^{-1}$ (relevant to the Atlantic storm track) and is negatively correlated at higher jet speeds (relevant to the Pacific). This is manifest most clearly as a midwinter minimum of the Pacific storm track, but is also evident on interannual time scales (Zhang and Held 1999; Chang 2001; Nakamura et al. 2002). While Nakamura's study mostly em-

Corresponding author address: Dr. Nili Harnik, Lamont-Doherty Earth Observatory, Columbia University, 61 Route 9W, Palisades, NY 10964.

E-mail: nili@1deo.columbia.edu phasized the seasonal time scales, Zhang and Held (1999) pointed out the distinction between interannual and seasonal time scales but hypothesized that the cause for a negative correlation is similar for both time scales. Chang (2001), on the other hand, found evidence that different mechanisms cause the relation for the two time scales, with changes in diabatic heating contributing strongly on seasonal time scales, and changes in eddy structure and evolution dominating the interannual time scales. In this study we concentrate on understanding the interannual-time-scale variability, by studying dynamical effects that might cause a negative correlation between storm track and jet strength. We will discuss the relevance of our results to the seasonal time scale in section 5.

Figure 1 shows the January mean jet and strom track variance, averaged over the seven years with strongest and weakest jets. It is clear that the spatial growth rate in the downstream direction in the storm track is stronger during the weak jet years. The cause for this relation 

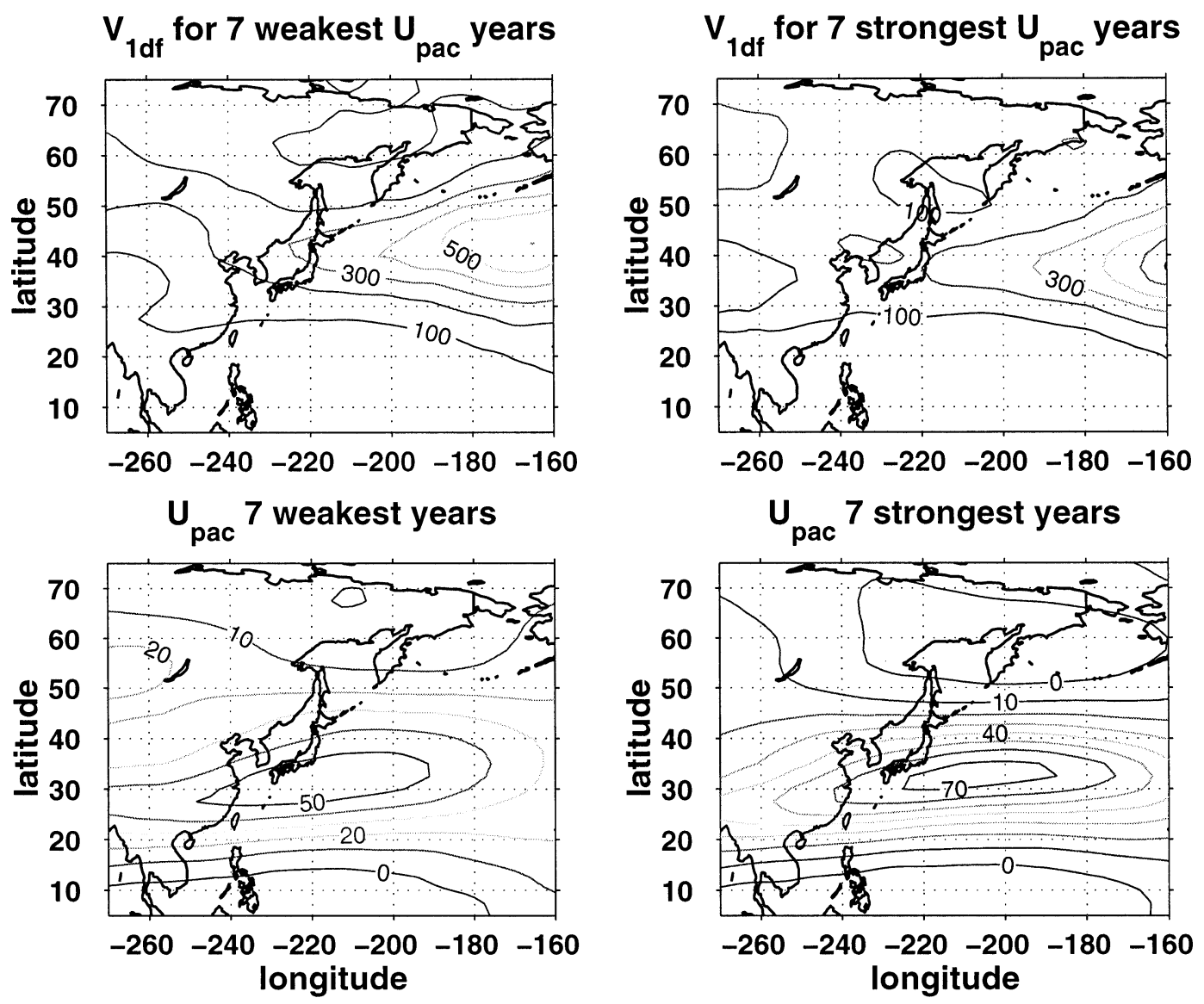

FIG. 1. (top) The storm track as represented by a 24-h difference-filtered meridional wind $\left(\mathrm{m}^{2} \mathrm{~s}^{-2}\right)$ averaged over the seven weakest (left) and strongest (right) jet years. (bottom) Same as top, only 300-hPa zonal wind (m s ${ }^{-1}$ ).

is not obvious since we expect eddies to grow faster, rather than slower, when the jet is strong. One simple explanation (suggested by Nakamura 1992) is that eddies move more quickly out of the storm track region when the jet is strong, allowing them less time to amplify. This increase in propagation speed, however, needs to overcome the increase in growth rate. Chang (2001) compared the energy budget of the storm tracks for Januaries with weak and strong jets, using GCM simulations and observations. He found that on interannual time scales dynamical effects were the most important for explaining the observed variability, with eddies being more top-trapped near the tropopause, propagating with a much higher group velocity, and being less efficient in tapping the surface baroclinicity when the jet is strong. This suggests that, when the jet is strong, eddies move faster out of the unstable region, as expected, but they also grow less efficiently, resulting in a weaker storm track. The changes in group velocity and growth rate seem to be related to the changes in the vertical structure of the eddies, but the exact mechanism is still unclear. In particular, as Chang (2001) pointed out, the simplest model for baroclinic instability, the Eady (1949) model, does not predict these changes.

This suggests that a linear model of baroclinic growth, which relates eddy growth to the shear in the center of the jet, may not be sufficient to explain the observed relation. It is possible, however, that a linear model that incorporates the meridional jet structure and variability will be able to reproduce behavior like the observed. To examine this we calculate the January mean zonal wind averaged over the Pacific jet region $\left(120^{\circ}-150^{\circ} \mathrm{E},{ }^{1}\right.$ referred to as $U_{\mathrm{PAC}}$ ), using National Centers for Environmental Prediction-National Centers for Atmospheric Research (NECP-NCAR) reanalysis for 1949-2001. The top plot in Fig. 2 shows the $300-\mathrm{hPa}$ wind profiles, centered about the jet maximum, for the strongest and weakest jet profiles, as well as the average over the strongest, middle, and weakest third of the years. The jet strength varies roughly between $50-76 \mathrm{~m} \mathrm{~s}^{-1}$, and

\footnotetext{
${ }^{1}$ The overall results are not sensitive to the range of longitude over which we average.
} 

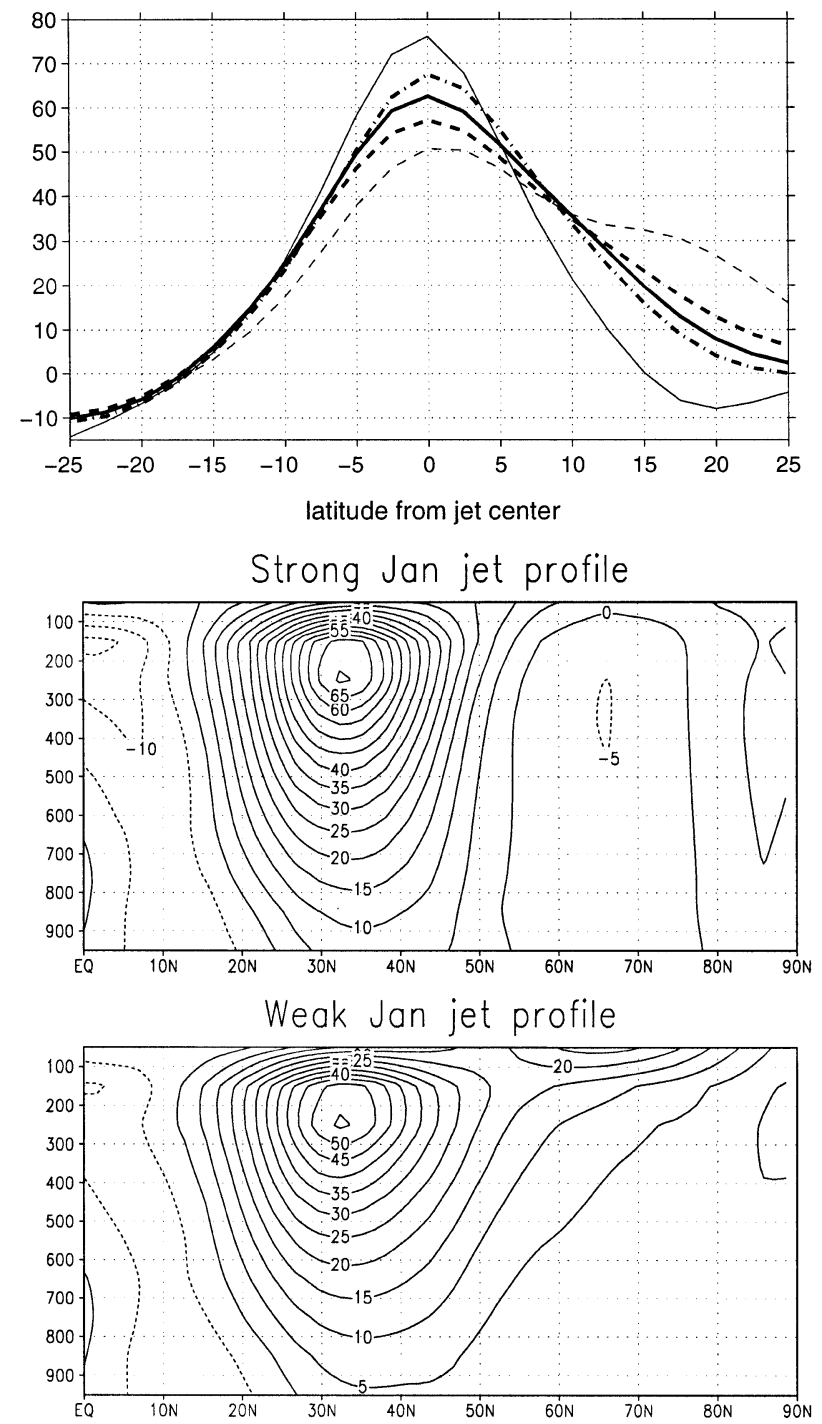

FIG. 2. (top) Latitudinal profile (centered around the jet maximum) of the strongest and weakest jets (thin solid and dashed lines, respectively), and the average over the strongest, middle, and weakest one-third of the jet profiles (thick dashed-dotted, solid and dashed lines, respectively). (middle and bottom) The zonal wind over the Pacific, averaged over the five strongest and weakest jet years (used in the primitive equation linear model runs, see text for details). Negative values dashed; contour interval is $5 \mathrm{~m} \mathrm{~s}^{-1}$.

there is a clear tendency of the jet to be narrower when it is strong.

The reason for the stronger jets being narrower is not clear, but there is some evidence that this is not due to feedback forcing from the local storm track eddies but rather forced externally. Nakamura et al. (2002) suggested that the changes in stationary wave could be associated with a wave train that appears to originate from the North Atlantic reaching into the far east, while Yin (2002) suggests that this could be the response to forcing by tropical convection based on an idealized GCM study. This is also consistent with the suggestion of Lee and Kim (2003) that the Pacific jet is externally driven (as opposed to the Atlantic jet which is eddy driven). In this study, we will assume that the observed jet strength-width relation is externally set and use this relation to constrain eddy evolution.

Regardless of the causes, we expect the variations in jet structure to affect wave growth. For example, the Eady model predicts that the growth rate of the most unstable normal mode decreases as the meridional wavenumber increases. One simple model of the storm tracks (Chang 2001) is of upper-level wave packets entering the storm track region from upstream and propagating along the jet as they amplify. This implies the existence of a waveguide that prevents the eddies from propagating away from the jet region in the meridional direction. This view, which has some support in the literature (e.g., Lee and Anderson 1996; Nakamura and Sampe 2002), implies that the meridional wavenumber of the perturbations is set by the waveguide, which is determined by the jet structure. Ioannou and Lindzen (1986) showed, using the WKB approximation, that growth of baroclinically unstable waves on a jet can be approximated to first order by waves growing on a vertically varying basic state taken from the jet center, with the meridional wavelength set by the meridional structure of the jet (a rough approximation is to have half a meridional wavelength equal to a half-width of the observed jet). We used a 3D primitive equation model to calculate the growth rate and structure of the most unstable modes on 2D (latitude-height) basic-state jets that we strengthen and narrow as observed, and the results (see section 3) support this interpretation. While this is not the only way in which meridional wind variations can affect wave growth, it is a straightforward direct effect on growth rate, which can easily be incorporated in a simple model.

In this paper we examine the effects of shear and meridional jet structure on the linear growth and propagation of waves in the context of storm tracks. We start by presenting a simple model of the storm tracks that we use as a framework for our model studies (section 2). We then present results from primitive equation and quasigeostrophic model runs in sections 3 and 4. In section 5 we discuss the relevance of our idealized calculations to the storm tracks and we conclude in section 6 .

\section{A simple model of spatial growth rate}

Spatial, rather than temporal growth, is the relevant quantity for storm tracks that are characterized by the time-mean eddy variance. Assuming storm tracks are seeded by upper-level waves coming into the storm track region from upstream (see section 5 for references), we study the spatial growth and evolution of an upper-level wave packet, which propagates along the jet stream and amplifies by inducing a surface perturbation that reinforces the initial upper-level wave (Hoskins et al. 1985). 
In section 5 we discuss the relevance of our results to real storm tracks.

We obtain the spatial growth rate using an equation for the eddy energy $E$ along the storm track ( $x$ direction). This derivation, which follows the heuristic formulation put forth by Swanson et al. (1997), is presented in Chang (2001) and is repeated here for clarity of the discussion:

$$
\frac{\partial E}{\partial t}+\boldsymbol{\nabla} \cdot \mathbf{F} \approx C_{g} \frac{\partial E}{\partial x}=\text { source }+ \text { sink }
$$

where $\mathbf{F}$ is the flux of eddy energy, which equals the eddy energy times a group velocity $C_{g}$, and we assume $C_{g}$ is constant in $x$ in our model.

In steady state, the eddy energy flux divergence balances the sources and sinks. We assume the source term can be expressed as a growth rate $\sigma$ times eddy energy and use baroclinic instability theory to estimate $\sigma$. For simplicity, we also assume damping is proportional to eddy energy (we relax this assumption later on):

$$
\begin{aligned}
\text { source } & \approx \sigma E, \\
\text { sink } & \approx-\gamma E .
\end{aligned}
$$

Substituting into Eq. (1) and taking a time average, we get an expression for the spatial growth of eddy energy $\left(S_{\mathrm{gr}}\right)$ :

$$
S_{\mathrm{gr}} \equiv \frac{1}{E} \frac{\partial E}{\partial x} \approx \frac{\sigma-\gamma}{C_{g}} .
$$

There are two competing effects of group velocity and growth rate. When the jet strengthens, the eddies move faster out of the unstable region, which tends to weaken $S_{\mathrm{gr}}$. On the other hand, when the jet is stronger, the local growth rate increases; causing $S_{\mathrm{gr}}$ to increase. Equation (4) suggests that it is the ratio of $\sigma$ and $c_{g}$ that matters. Which effect dominates depends on which of the two varies more with jet strength.

\section{Stability analysis of Pacific jet profiles using a primitive equation model}

The model used is based on the dynamic core of the Geophysical Fluid Dynamics Laboratory (GFDL) climate model (see Held and Suarez 1994). The integrations are performed with 10 evenly distributed sigma levels, at a horizontal resolution of T42 (64 Gaussian grid points in the meridional direction). The basic-state temperature and wind are a zonal mean across the Pacific storm track entrance region $\left(120^{\circ} \mathrm{E}-180^{\circ}\right)$, taken from the NCEP-NCAR reanalysis data for different years. The resulting basic states (shown in Fig. 2, middle and bottom panels), which are 2D, represent strong and weak jet years in the Pacific. ${ }^{2}$ We see that, indeed, the jet is stronger but narrower during the strong jet years. Note

\footnotetext{
2 The strong jet years included in the composite are 1970, 1977, 1981, 1985, and 1988, while the weak jet years are 1971, 1972, 1973, 1989, and 1993.
}

TABLE 1. The zonal wavenumber, growth rate, group velocity, and implied spatial growth rate (the temporal growth rate divided by the group velocity) of the most unstable linear modes for the different runs of the $3 \mathrm{D}$ primitive equation model (see text).

\begin{tabular}{llcccc}
\hline \hline Jet speed & Friction & $\begin{array}{c}\text { Wave- } \\
\text { number }\end{array}$ & $\begin{array}{c}\text { Growth } \\
\text { rate }\end{array}$ & $\begin{array}{c}\text { Group } \\
\text { velocity }\end{array}$ & $\begin{array}{c}\text { Implied } \\
\text { spatial } \\
\text { growth rate }\end{array}$ \\
\hline Strong & Weak & 7 & 0.47 & 16.3 & 0.34 \\
& Medium & 7 & 0.36 & 16.0 & 0.26 \\
& Strong & 6 & 0.20 & 16.3 & 0.14 \\
Weak & Weak & 7 & 0.39 & 11.7 & 0.39 \\
& Medium & 7 & 0.29 & 11.3 & 0.30 \\
& Strong & 6 & 0.13 & 11.5 & 0.13 \\
Weak*1.3 & Weak & 7 & 0.55 & 15.9 & 0.40 \\
& Medium & 7 & 0.43 & 15.1 & 0.33 \\
& Strong & 6 & 0.28 & 14.1 & 0.23 \\
\hline
\end{tabular}

that the maximum speed of the strong jet is 1.3 times that of the weak jet.

The model is dry, with a 30-day Newtonian cooling in the free atmosphere, reaching 2 days in the lowest sigma level. As discussed in Valdes and Hoskins (1988), the growth rate and structure of the unstable modes are not very sensitive to the Newtonian damping. Surface friction is represented by Rayleigh friction with a damping time scale of 2,1 , and 0.5 days for the weak, medium, and strong friction experiments, respectively. As in previous work (e.g., Farrell 1985; Valdes and Hoskins 1988), the growth rates are very sensitive to the strength of surface friction.

For each basic state and zonal wavenumber, a small perturbation is introduced and the model integrated. Nonlinearity is suppressed by rescaling the perturbation once its amplitude (in terms of vorticity perturbation) reaches $1 \%$ that of the basic state. The experiment is run until the growth rate and wave structure become steady.

The zonal wavenumber, growth rate, group velocity, and implied spatial growth rate (the temporal growth rate divided by the group velocity) for the different cases are tabulated in Table 1. Here, the group velocity is estimated by finite differencing the wave frequency by the zonal wavenumber between the two adjacent fastest growing normal modes. Comparing the strong and weak jet cases, we see that for all three friction values, the temporal growth rate is higher for the strong jet, but for the weak and medium friction cases, the increase in growth rate is less than that implied by the change in the jet speed (30\%). The group velocity for all cases is higher for the strong jet runs by more than $30 \%$. The implied spatial growth rate is therefore higher for the weak jets except for the strong friction runs (especially the weak jet) for which the growth rate is strongly suppressed by surface friction.

The structure of the most unstable mode for both jets and medium friction are shown in Fig. 3. Shown are rms $v$ at $250 \mathrm{hPa}$ (normalized to unit amplitude at the 
a) Model $250 \mathrm{hPa} \mathrm{v}$
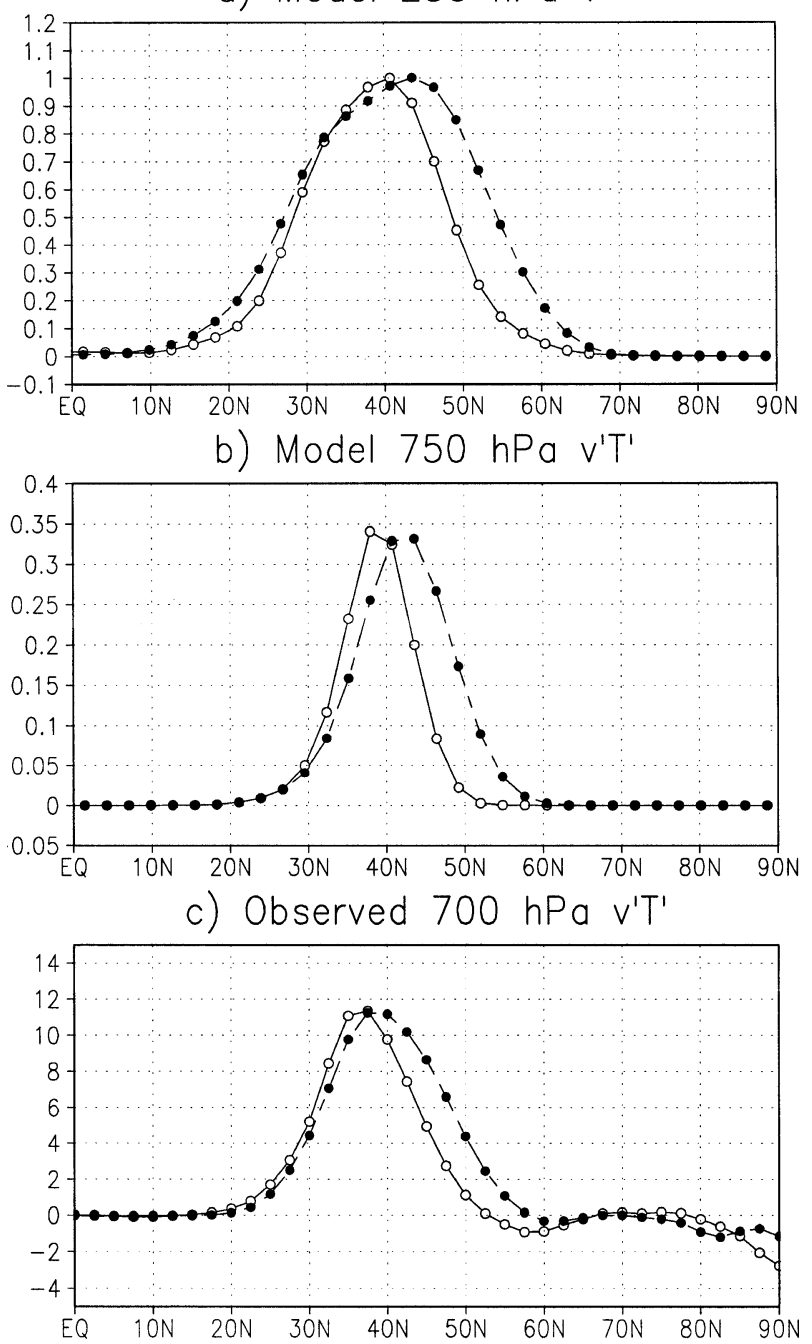

FIG. 3. (a) The latitude variation of the meridional wind anomaly at $250 \mathrm{hPa}$ and (b) heat flux at $750 \mathrm{hPa}$ of the most unstable normal modes of the primitive equation model runs, and (c) the observed 700-hPa Jan heat fluxes for the strong (solid with open circles) and weak (dashed dots) jets of Fig. 2. In (a) and (b) the meridional winds and heat fluxes are normalized such that the maximum 250-hPa $v$ equals $1 \mathrm{~m} \mathrm{~s}^{-1}$ for both cases. In (c) the heat flux for the strong jet is multiplied by 1.4 to facilitate the comparison of meridional structure.

peak, plot a) and poleward heat flux at $750 \mathrm{hPa}$ (also normalized, plot b). Clearly, the most unstable mode for the narrower jet has a significantly narrower structure. In plot c, we show the meridional profile of the observed bandpass-filtered 700-hPa poleward heat flux averaged between $120^{\circ} \mathrm{E}$ and $180^{\circ}$, taken from the strong jet and weak jet years. The results show that the observed heat flux is indeed narrower during the strong jet years. However, the correlation between the meridional width of 700 -hPa heat flux and jet strength is quite weak $(-0.25$, which is only significant at the $90 \%$ level but not at the $95 \%$ level) for the entire 51 years of reanalysis data.
To show that this behavior of the spatial growth rate is indeed due to the change in jet profile, we generate a new jet profile by increasing the strength of the weak jet by 1.3 times while maintaining its latitudinal profile so that its structure is of the weak jet, while its maximum strength is of the strong jet. The results for this case are also shown in Table 1. Compared to the weak jet case, we see that the temporal growth rate increases by more than $30 \%$, which is the amount by which the jet strength increased. This is due to the effect of surface friction, as expected from Eq. (4) based on the arguments in Chang (2001). The resulting spatial growth rate is larger for the weak times 1.3 jet than for the weak jet, even though the group velocity has increased. This shows that simply increasing the advection out of the region is not sufficient to suppress spatial growth. We must take into account both the effects of an increase in the group velocity due to the increase in jet speed, as well as the reduction in increase of temporal growth rate due to the narrowing of jet, in order to explain the decrease in spatial growth rate for the strong jet case.

These model runs suggest that the variations in meridional confinement may explain the observed reduction in the rate at which temporal growth rate increases with shear. In the next section we use a much simpler and numerically cheaper model to examine in detail how the jet structure affects temporal and spatial growth rate, group velocity, and vertical wave structure for various types of basic-state and model parameters.

\section{Linear quasigeostrophic model results with variations in jet width taken into account}

We use a simple linear quasigeostrophic $\beta$-plane model of baroclinic instability, with different jet profiles, to perform two kinds of calculations. The first is an eigenvalue calculation for the vertical structure, growth rate, and phase speed of the corresponding baroclinically unstable normal modes for a range of zonal wavenumbers. The second is a time-dependent integration of our model, starting from an upper-level wave packet, centered around a specified zonal wavenumber $k_{o}$. We evaluate the spatial growth rate from $S_{\mathrm{gr}}=\sigma^{*} / C_{g}$, where $\sigma^{*}$ is the effective growth rate of perturbations in the model, including the effect of damping. The basic-state and model setup is similar to Harnik and Lindzen (1998) and the numerical solution method is similar to Farrell (1982), where we decompose the equations into zonal Fourier components, and for each zonal wavenumber we solve the vertical eigenvalue problem, project the initial perturbation on the eigenvectors, and sum their contributions to obtain the time-dependent solution.

We incorporate the observed relation that the jet narrows as it strengthens into our model by assuming the jet width determines the meridional structure of the eddies and specify the meridional wavenumber accordingly. The validity of this approach is supported by the 
results of Ioannou and Lindzen (1986), as well as the results of section 3 .

The model basic-state wind and temperature have a troposphere and stratosphere, and the density decreases exponentially with height. Our channel is centered at $40^{\circ}$ latitude. The top boundary is a lid at around $22 \mathrm{~km}$, and we have a sponge layer that starts at around $15 \mathrm{~km}$, to absorb any wave activity that propagates to these heights. ${ }^{3}$ We have 70 grid points in the vertical. There are various ways to specify the tropospheric shear, which differ in the resulting meridional gradient of potential vorticity (PV). The simplest is to specify a constant shear with height, and let the PV gradient increase with shear [a Charney model (Charney 1947)]. A different approach is to hold the meridional PV gradient fixed, while we increase shear by adding vertical curvature to the wind profile [an Eady-like model (see Lindzen 1994; Harnik and Lindzen 1998)]. The value of meridional PV gradients makes a big difference for the growth of short waves. In the Charney model, baroclinic growth occurs through an interaction between surface edge waves and internal Rossby waves. In the modified Eady model [which differs from the Eady model by being on a $\beta$ plane (Lindzen 1994)] there are no internal tropospheric waves because the meridional gradients of PV are zero. Instead, the model supports tropopause waves, which interact with the surface waves and grow, provided these waves are deep enough. Since the vertical scale is proportional to the horizontal scale of the waves, short waves are stable. As a result, one of the main differences between the Eady and Charney models is that short waves are neutral in the former and unstable in the latter. This is relevant to our calculations because the decrease of meridional wavelength with shear pushes the model to the short-wave regime.

Unless otherwise specified, in all the following runs we increase the meridional wavenumber as we increase shear. Figure 4 shows the relation between meridional wavenumber and tropopause wind in our model, plotted with the observed relation between the maximum jet speed, using 300-mb zonal winds averaged over the Pacific $\left(120^{\circ}-150^{\circ} \mathrm{E}\right)$, and the distance between the latitudes at which the winds reach half their peak value. We use a simple linear fit to the observed jet strengthjet width relation, and the rough approximation that half a meridional wavelength equals the half-width of the observed jet, so that the meridional width in our model varies between a meridional half-width of $36^{\circ}$ (equivalent to a zonal wavenumber of 3.8 in units of number of waves that fit into a latitude circle) for a jet of around $22 \mathrm{~m} \mathrm{~s}^{-1}$, to $15^{\circ}$ (zonal wavenumber 9.4) for a jet of $77 \mathrm{~m} \mathrm{~s}^{-1}$. Observations lie along the strongest three or four jet profiles. An alternative to the linear fit is to assume that the meridional temperature difference be-

\footnotetext{
${ }^{3}$ Since we are interested mostly in medium and short waves, which do not propagate up to the stratosphere, the waves hardly feel the sponge layer and lid.
}

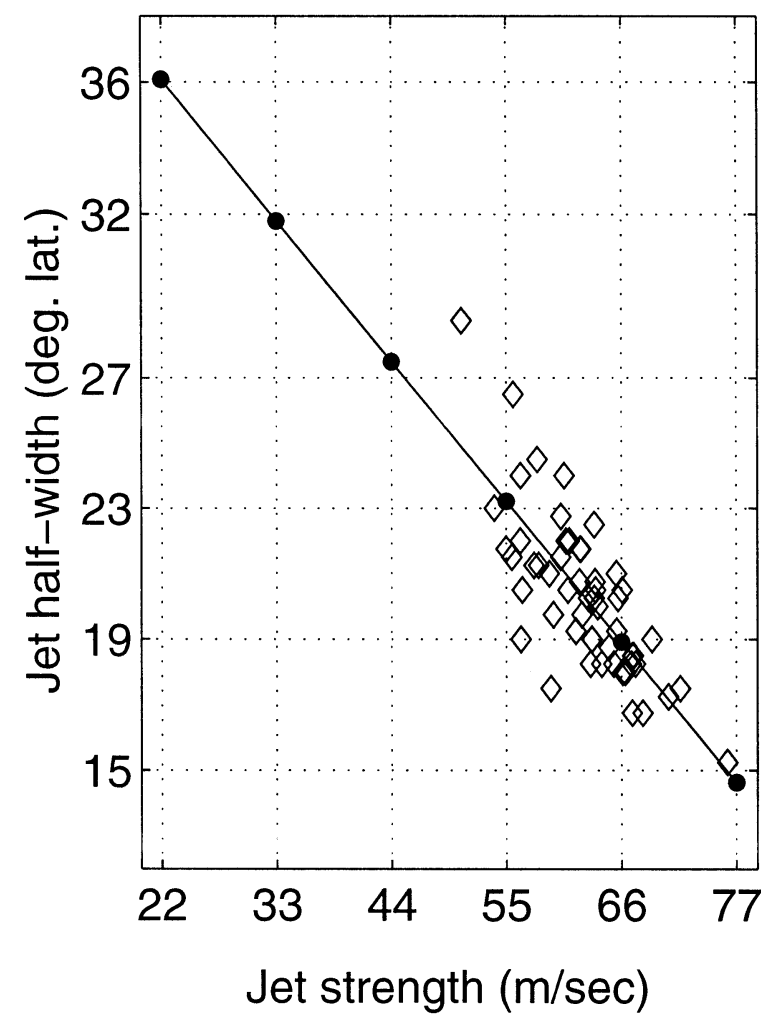

FIG. 4. The relation between the jet strength $\left(\mathrm{m} \mathrm{s}^{-1}\right)$ at model tropopause and at $300 \mathrm{hPa}$ for observations and the jet half-width (in ${ }^{\circ}$ lat) for model (line with solid circles) and observed (diamonds).

tween the northern and southern boundaries is fixed (corresponding roughly to fixed zonal transport due to the thermal wind relationship). This fits the observations just as well, with the main difference being over the weak jet regime. The results presented below are not sensitive to the specific jet strength-width relation used, provided it roughly captures the observed relation.

\section{a. Charney/Green-type basic state}

First, we examine results based on the Charney profiles, with no damping. Figures 5a,b show the basic state for a set of runs in which the wind is specified to have a constant shear in the troposphere and zero shear in the stratosphere. The tropospheric wind (below about 9 $\mathrm{km}$ ) increases from $3 \mathrm{~m} \mathrm{~s}^{-1}$ at the surface with vertical shear between $2.5-8.6 \mathrm{~m} \mathrm{~s}^{-1} \mathrm{~km}^{-1}$ (runs 1-6, respectively). Also shown (Fig. 5b) is the Brunt-Väisälä frequency, which is the same for all runs, and the corresponding profiles of meridional PV gradients, which increase with shear in the troposphere between $2.4-5.8$ $\beta$ (for runs $1-6$, respectively).

Figure $5 \mathrm{c}$ shows the normal-mode growth rate for the six basic states. We see that, for the most unstable wavenumbers (zonal wavenumber $<11$ ), the growth rate increases with jet strength for runs $1-5$ and decreases 

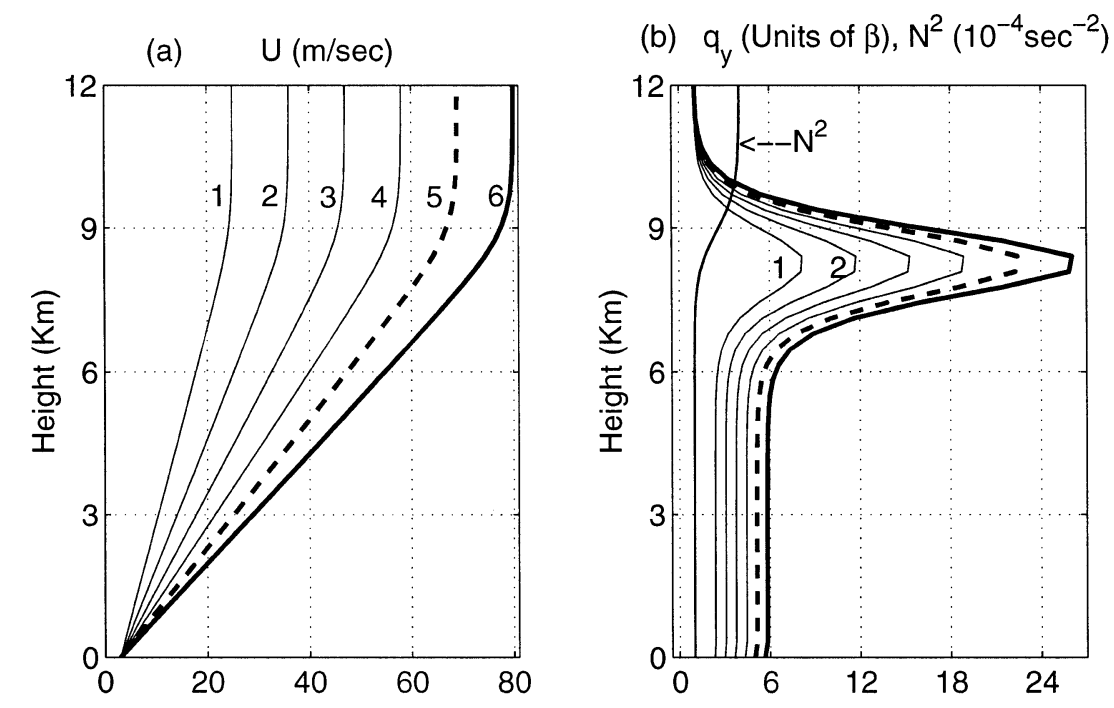

(c) Growth rate $\left(\right.$ day $\left.^{-1}\right)$



(d) Spatial growth rate $(1 / 1000 \mathrm{~km})$

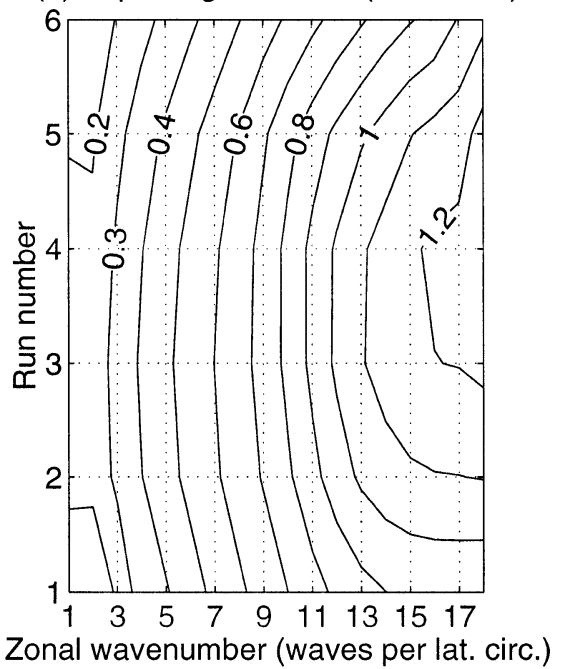

FIG. 5. (a) Zonal mean wind ( $\mathrm{m} \mathrm{s}^{-1}$ ), (b) the meridional gradient of PV (units of $\beta$ ), (c) the normal-mode growth rates $\left(\mathrm{day}^{-1}\right)$, and (d) the corresponding spatial growth rate $\left(10^{-6} \mathrm{~m}^{-1}\right)$ as a function of zonal wavenumber and nondimensional shear (used to number the runs), for the six basic states used in the model run. The nondimensional shear (run number) is marked in (a)-(c); thick line is run 6; thick dashed is run 5. Also shown in (b) is the Brunt-Väisälä frequency $\left(10^{-4}\right.$ $\left.\mathrm{s}^{-2}\right)$.

between runs 5 and 6 . Dividing by the group velocity (which increases slower than linearly with shear), we get a spatial growth rate that increases, then decreases with shear, with the transition occurring at runs 3 or 4 (Fig. 5d). Our model therefore seems to account not only for the decrease of storm track strength with shear, but also for the fact that the decrease is only observed for large shears.

To understand this transition, we calculate the growth rate for a range of meridional wavenumbers and shear for zonal wavenumber 8 (results for other zonal wavenumbers are similar). The results are shown in Fig. 6. If the meridional wavenumber is kept constant as the shear is increased (moving up from curve 1 to 2 to 3 , etc., in Fig. 6), the growth rate increases with increasing shear, as expected, but the increase is fastest for smaller meridional wavenumbers. If we keep the shear the same but increase the meridional wavenumber (stay on the same curve but migrate to the right), the growth rate generally decreases with increasing meridional wave- 


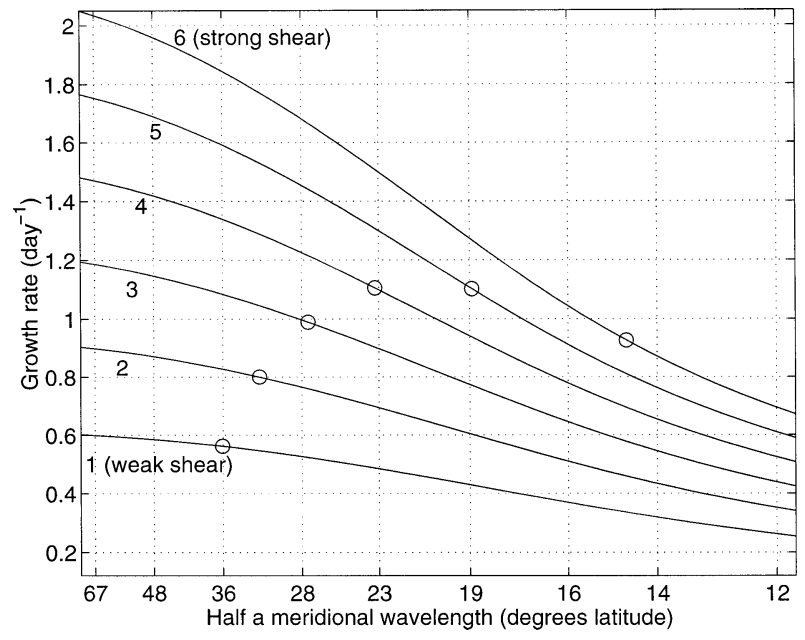

FIG. 6. The normal-mode growth rate $\left(\right.$ day $\left.^{-1}\right)$ as a function of meridional wavenumber for zonal wavenumber 8 . Curves are for the different basic states of Fig. 5 (numbered). The meridional wavenumbers used for each run and the corresponding growth rate are marked by the circles.

number, ${ }^{4}$ with the decrease fastest for large shear and large wavenumbers. Combining the two effects, as the shear increases and jet width decreases, we move from one curve to the next while shifting toward the right (following the trajectory of the circles shown in Fig. $6)$. We see that the shear effect wins for small shears and wavenumbers and the wavenumber effects wins for large shears and wavenumbers, resulting in the two regimes.

This behavior also holds for the time-dependent model. We start with an upper tropospheric wave packet centered around zonal wavenumber 8.5 (using other wavenumbers yields similar results) and let it evolve on each of the six basic states. Figure 7 a shows the initial tropopause wave packet geopotential height, and Figs. $7 b-f$ show the longitude-time evolution of the wavepacket envelope at $9 \mathrm{~km}$ for runs 4 and 6 and the timeheight structure of the envelope at the center of the wave packet for runs 2, 4, and 6 . We calculate the envelope by interpolating between maxima of the absolute value of the wave geopotential height field. It is clear, by looking at a specific envelope value for each of the runs (e.g., the dashed lines), that the temporal and spatial growth rates are largest for intermediate shear values (the dashed line appears earlier and at a smaller longitude in run 4, compared to runs 2 and 6).

We see two stages in the evolution of the wave packet. During the initial stage the vertical structure of the wave packet changes as it spins up a surface circulation (e.g., days $0-3$ of run 2 , Fig. $7 b$ ), while in the second stage

\footnotetext{
${ }^{4}$ For the very long waves (planetary scales), the growth rate increases with wavenumber. Given the horizontal scales that are relevant to the storm tracks, this is not relevant here.
}

the vertical structure is fixed in time with the amplitude increasing exponentially in time (e.g., after day 4 of run 2 ), as is the case with normal modes. Estimating a growth rate and group velocity from the amplitude and longitude of the center of the wave packet, respectively, we find that the group velocity and growth rate also vary in the initial stage, reaching a constant value in the second stage, which we verify to be equal to the values obtained from the normal-mode calculation for zonal wavenumber 8.5 (Fig. 5c). We therefore refer to two stages as the nonmodal and normal-mode growth stages, respectively. We find that, as shear is increased, it takes the waves less time to reach the normal-mode stage.

Figure 8 a shows the group velocity divided by shear, for each of the six runs, estimated from the slope of a linear fit to the longitude of the tropopause wave packet center for different time periods, representing the initial nonmodal stage (days $0-3.3$ ) and the normal-mode stage (days 18.6-25). We also use days 0-7 to represent a characteristic storm track value, which may comprise a mix of both stages. We see that the group velocity is larger in the initial stage (thin dashed) compared to the normal-mode stage (solid) for runs 1-3. For runs 4-6, the normal-mode stage is reached in less than 3 days. We also see that, contrary to observations, the ratio of group velocity to shear decreases with shear at all stages of wave growth (the group velocity itself increases with shear).

\section{b. Eady-like basic state}

The vertical structure of the wave packets shown above is largest at the surface, unlike the observed wave packets, which have a peak at the tropopause with a secondary peak near the surface. This is expected based on the normal modes of the Charney model. Waves in the Eady model, on the other hand, do have a peak at the tropopause..$^{5}$

Figures 9a,b show the zonal mean wind and meridional PV gradients for the corresponding set of runs with the Eady-like basic states. The Brunt-Väisälä frequency is the same as in Fig. 5b. The wind is specified so that the mean tropospheric shears are the same as in Fig. $5 \mathrm{a}$ and the shear is zero above about $10 \mathrm{~km}$. The change in shear with height, along with the increase in the Brunt-Väisälä frequency from tropospheric to stratospheric values, result in having a peak of PV gradients at the tropopause. Note that a relatively small difference in the wind profiles in Figs. 9 and 5 results in a significant difference in the PV gradient in the troposphere. The PV gradients at the tropopause are also much larger due to the sharper change in vertical wind

\footnotetext{
${ }^{5}$ In the classical Eady model, there is complete symmetry about the midtroposphere, but in the modified Eady model $\left(\beta \neq 0\right.$, but $q_{y}$ $=0$ ) waves are larger at the tropopause.
} 
(a) Initial wavepacket

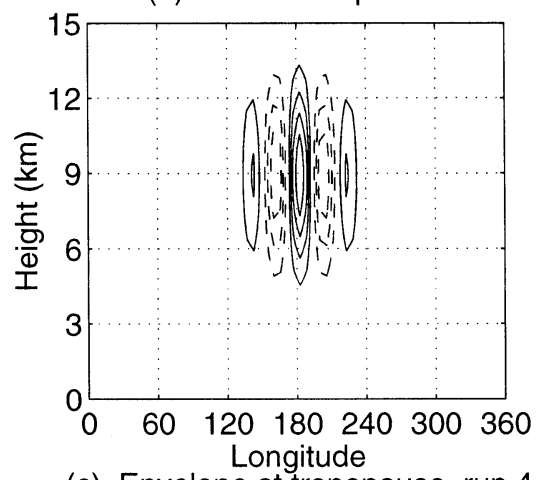

(c) Envelope at tropopause, run 4

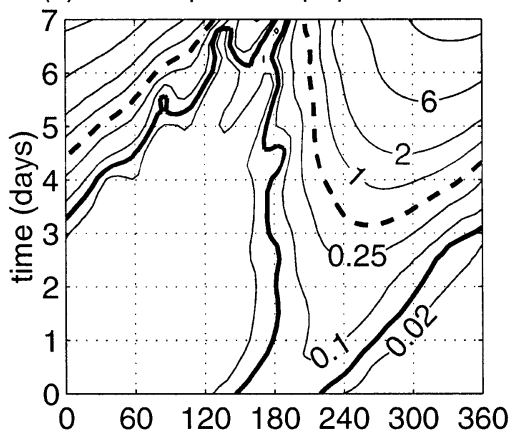

(e)

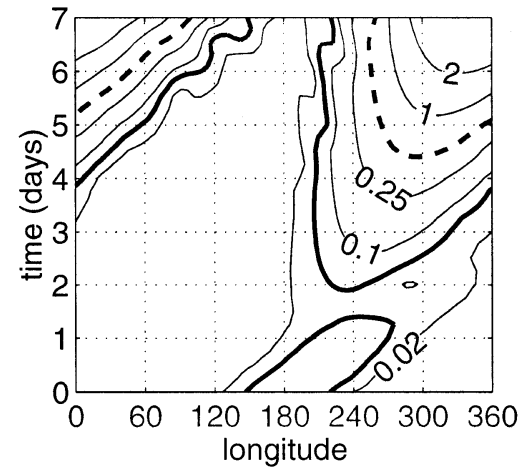

(b) Amplitude of envelope center, run 2



(d)
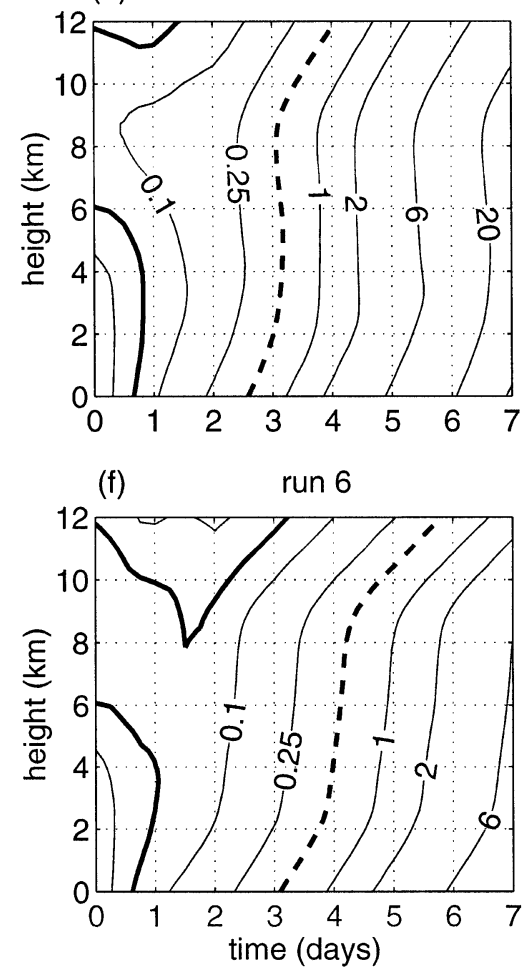

FIG. 7. (a) Longitude-height structure of the initial wave packet geopotential height. (b), (d), (f) Time-height plots of the amplitude of the wave packet envelope at wave packet center for runs 2, 4, and 6 of the Charney-type model. (c), (e) Longitude-time plots at the tropopause of wave packet amplitude for runs 4 and 6 . Line types are the same in plots (b)-(f).

shear with height near the tropopause. The meridional wavenumber is varied with shear as in Fig. 4.

The corresponding normal-mode growth rates for the case with no damping (Fig. 9c) increase, then decrease with shear, as in the Charney basic states, but there is an additional effect on growth rate of shifting the shortwave cutoff to smaller zonal wavenumbers (from zonal wavenumber 10 in run 1 to zonal wavenumber 4 in run 6). In our runs, this can have a very large effect on the evolution of initial upper-level wave packets of zonal wavenumbers that are close to the short-wave cutoff. The implied spatial growth rate is shown in Fig. 9d. Again, we see that for fixed zonal wavenumber, the spatial growth rate first increases with shear but then decreases as the jet gets too narrow, similar to the Charney case, but for this profile, since short waves are stabilized, the maximum spatial growth rate occurs when $k=8$ instead of at much shorter waves.

Figure 10 shows the envelope structure for runs 2, 4, and 5 of Fig. 9 starting from the same initial upper-level wavepacket perturbation of Fig. 7a. The increase, then decrease, of growth rate with shear is evident. The main difference compared to the results from previous runs is the short-wave cutoff shifting enough toward the long waves so that the wavenumbers in the initial wavepacket are stable. In our case the initial wave packet is essen- 
(a) Charney model

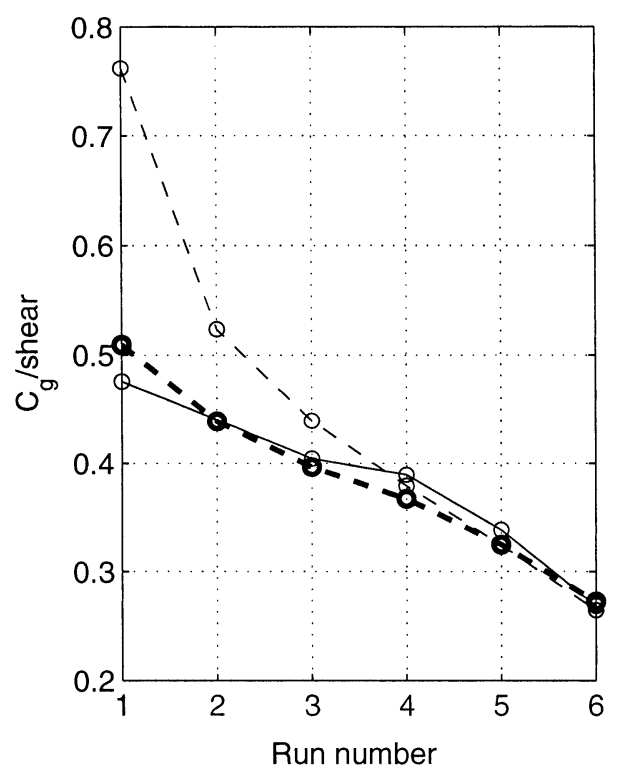

(b) Eady model

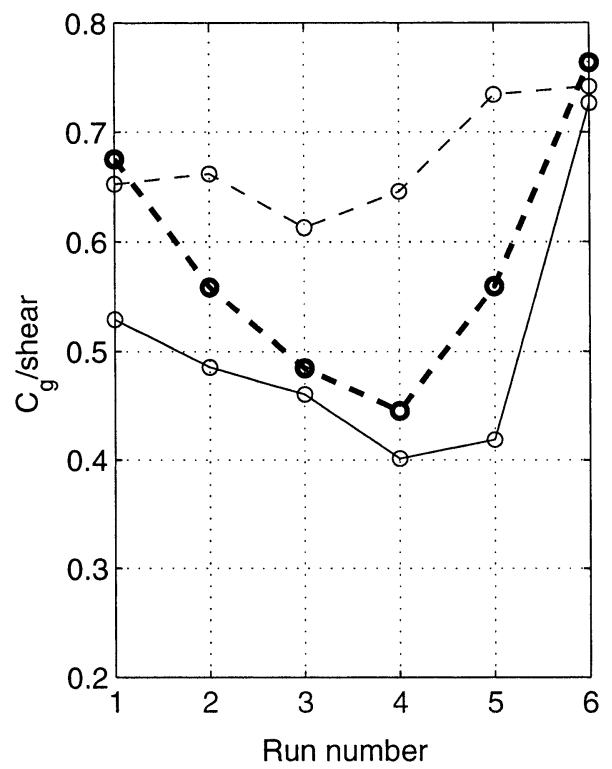

FIG. 8. The group velocity divided by shear, estimated from days 0 to 3.3 (thin dashed), 0 to 7 (thick dashed), and 18.6 to 25 (solid) of the time-dependent model solutions, for the six runs of the (a) Charney and (b) Eady models. Results are in nondimensional model units.

tially stable in run 6 and marginally unstable in run 5 . As a result, the vertical structure of the wave packet remains an upper-level wave packet for a relatively long time. ${ }^{6}$ The persistence of upper-level waves occurs because the model supports normal modes with such a structure-in the stable regime of the Eady model, the normal modes are upper level ${ }^{7}$ (see Rivest et al. 1992).

When the model is stable, the differences between the initial nonmodal stage and the normal-mode stage are very small. In the unstable runs, on the other hand, the initial wave packets have a more upper-level vertical structure and a faster group speed than the normal modes which are slowed down by the induced surface component. As a result, the transition of the model from an unstable to a stable regime as shear is increased involves large changes in the wave growth rate and group velocity. This is clear in Fig. 8b, which shows the group velocity divided by shear, for the initial and normalmode stages. The two are similar for run 6 , while for runs $1-5$ the group velocity is larger in the initial stage. Looking at the 0-7-day estimate (thick dashed line) we see that the group velocity increases faster than linearly with shear between runs $4-6$, similar to the observed behavior in the Pacific storm track entrance (Chang 2001). We note that, since in observations we average

\footnotetext{
${ }^{6}$ Even though the normal modes may eventually emerge, they will not be relevant for storm tracks if the perturbations leave the unstable region (which has a finite longitudinal extent) fast enough.

${ }^{7}$ There is actually a pair of normal modes for each wavenumbera surface edge wave and a tropopause wave.
}

over many eddies, we expect the transition from the stable to unstable regime to appear to be less abrupt than in our model.

Our results suggest that the transition of the Eadylike model to a state where upper-level wave packets are essentially neutral yields wave behavior that is closer to the observed, compared with the Charney-like basic state. This, however, might not hold under more realistic conditions. The neutrality of short waves in the Eady model depends on the meridional gradients of PV being exactly zero, but in reality we expect some gradients. Observations suggest that the PV gradients on isentropes are somewhere in between the Charney and Eady models, with gradients being on the order of $\beta$ in the troposphere (e.g., Kirk-Davidoff and Lindzen 2000). Repeating our runs, however, with $q_{y}=\beta$ instead of zero in the troposphere, we find that the main results are the same. The short waves are not neutral but they grow very slowly (much slower than the medium-scale waves), and the transition to the short-wave regime occurs at larger zonal wavenumbers for larger shears. As in the Eady-like runs, for strong shears, the normalmode stage is reached at a later time and the wave structure during the initial stage is much more upper level. The similarity to the Eady-type model is not surprising because when shear is constant with height (as in the Charney model), $\bar{q}_{y}$ is much larger than $\beta$ for strong jets (Fig. 5). Constraining the tropospheric PV gradients to be $\beta$ results, for strong jets, in a very large peak of PV gradients at the tropopause. This shifts the dynamics of the normal modes to a tropopause-surface 

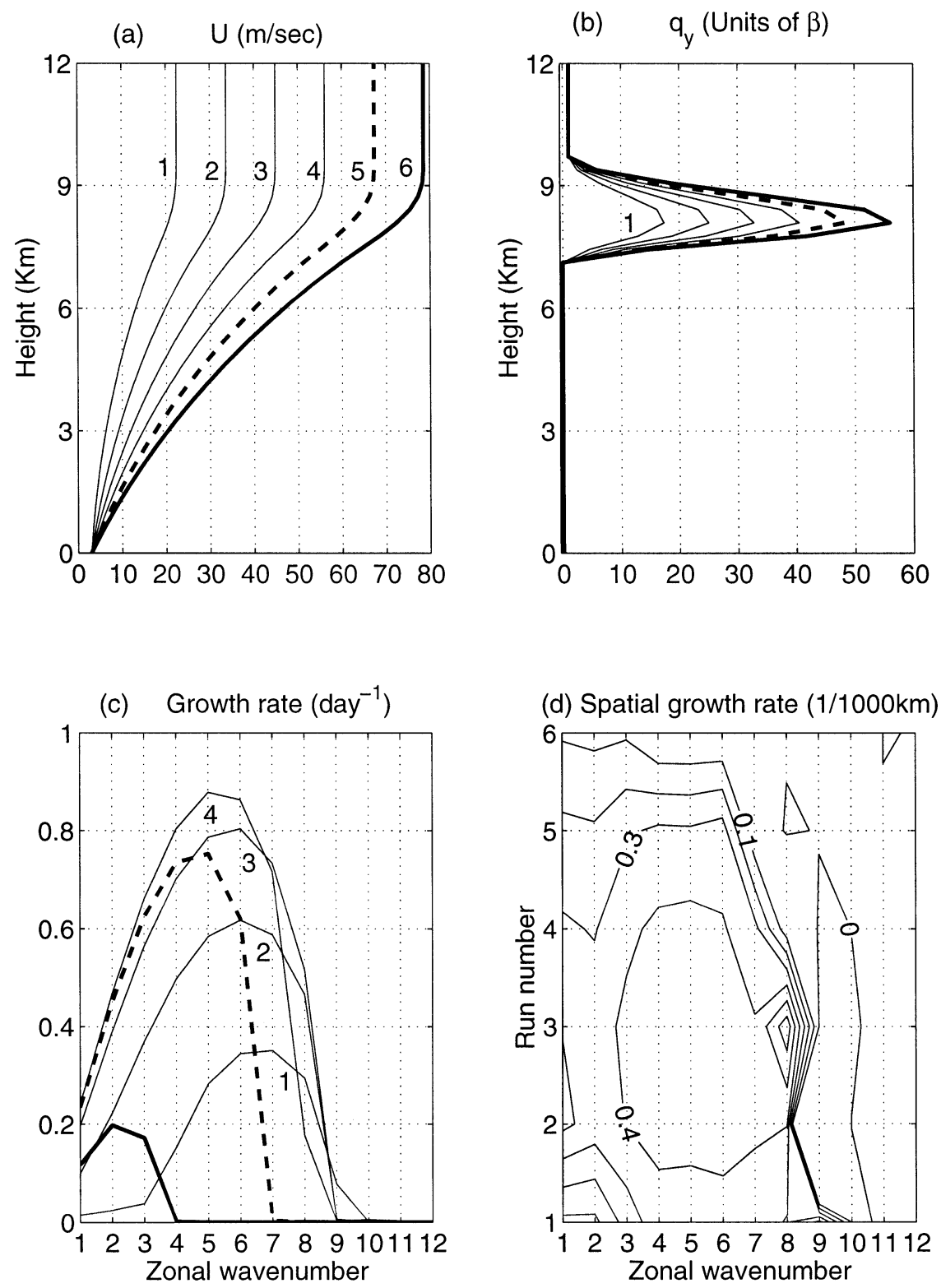

FIG. 9. As in Fig. 5 [without the Brunt-Väisälä curve in (b)] but for the Eady-type run.

interaction (as in the Eady model) rather than a troposphere-surface interaction as in the Charney model. For the observed type of behavior, we need the upper-level wavepacket to persist for a sufficiently long time. Rivest and Farrell (1992) showed that the pair of neutral shortwave modes in the Eady model transform to a decaying tropopause wave and an unstable surface wave, when a small $\bar{q}_{y}$ is added to the model. Moreover, for small values of $\bar{q}_{y}$, the slightly decaying tropopause waves, which they referred to as "quasi modes," have a decay rate that is proportional to $\bar{q}_{y}$, allowing them to be quite persistent in a time-dependent model.

A relevant study by Zurita and Lindzen (2001) suggests that, in both the Charney and the $\bar{q}_{y}=\beta$ models, short waves can be neutralized if the PV gradients are wiped out in the vicinity of their critical surface. It is very likely that such a neutralization will occur quite rapidly during the wave life cycle since wave-mean flow interaction is strongest at the critical surface. Moreover, the analysis of Zurita and Lindzen (2001) suggests this neutralization will happen at larger zonal wavenumbers in the strong/narrow jet runs. These neutralized waves, however, have the characteristics of lower-tropospheric rather than upper-level waves; therefore we expect the Eady-like model to better explain the observed group velocity and vertical structure dependence. Nonetheless, this neutralization mechanism can enhance the models' transition to a stable regime in the $q_{y}=\beta$ runs. 
(a) Envelope at tropopause, run 2

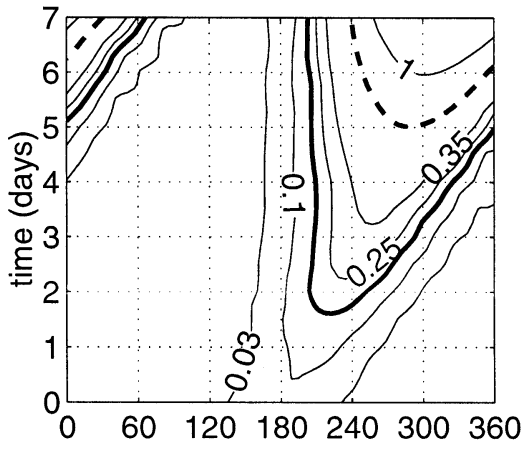

(c)

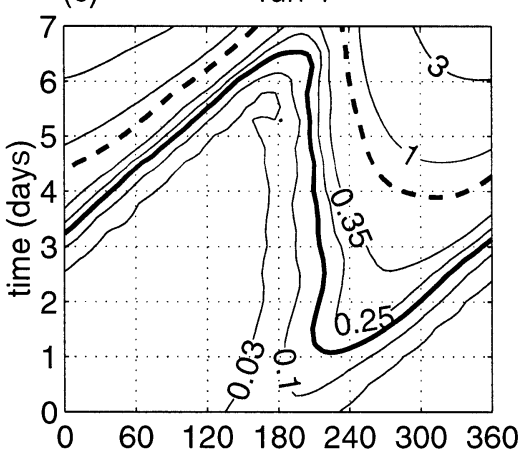

(e)

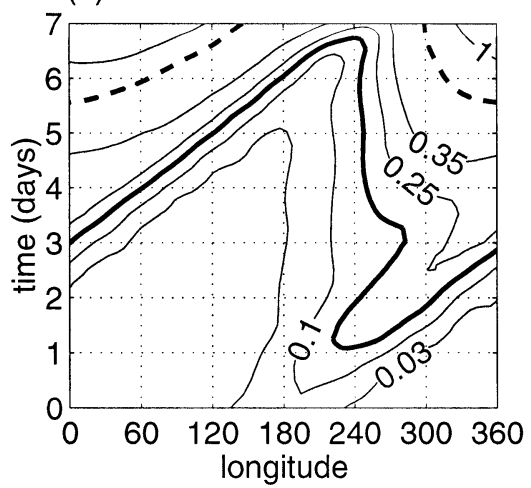

(b) Envelope center amplitude, run 2

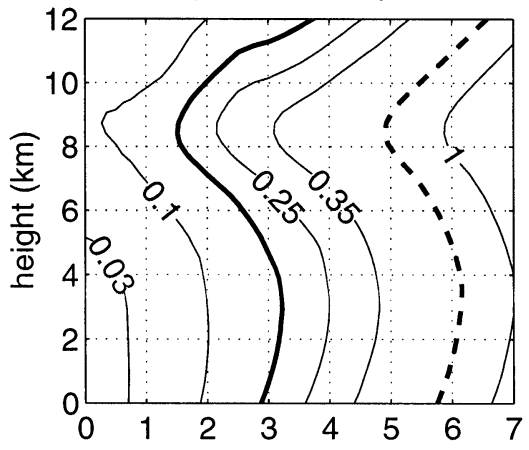

(d)
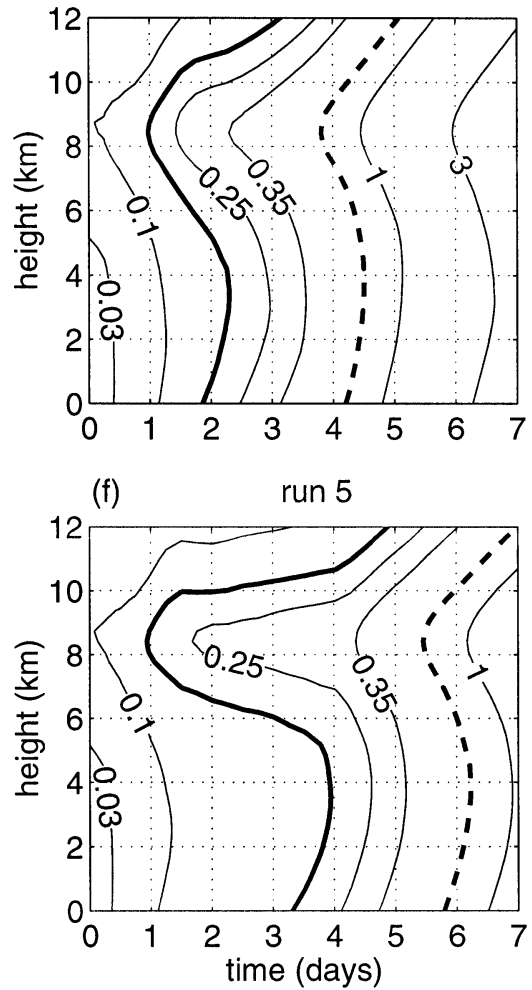

FIG. 10. The time evolution of wave packet envelope in the Eady-like model for runs 2, 4, and 5 (top to bottom): (a), (c), (e) as in Fig. 7c; (b), (d), (f) as in Fig. 7b.

In the appendix we describe the results of adding various forms of linear damping that are relevant to the storm track entrance regions. We find that damping does not change the main results of this study.

We also verify that the variations in meridional wavenumber are the key factor in yielding the observed type of behavior in our model runs by repeating the calculations for all basic states, keeping the meridional wavenumber constant. We find that both the growth rate and the spatial growth rate increase with shear for all wavenumbers. This suggests that the narrowing of the jet is crucial for the weakening of growth rate with shear. Moreover, without it, we expect an increase of the storm tracks with shear. We have repeated our runs with var- ious combinations of a different value for the meridional wavenumber, midchannel latitude, top boundary condition, $\beta$, different profiles for the wind and BruntVäisälä frequency, and a constant density with height. The results suggest that the increase of storm track strength with shear (when the jet width is kept fixed) for the inviscid case is mostly due to the effect of beta, with other factors having little effect.

\section{Discussion and the relevance to storm tracks}

In the previous section we showed that the narrowing of the jet as it strengthens results in a decrease in temporal as well as spatial growth rate. In addition, we 
showed that, if we have an Eady-type basic state, the narrowing also causes the model to be stable to the initial upper-level perturbation because the waves are too shallow to induce a surface component. As a result, the group velocity increases more than the increase of shear would imply, and the perturbations become more upper level for large and narrow jets. While this is similar to the observed relation, the relevance of our results to the real world is not obvious given the simplicity of our model. In this section we discuss the relevance of our idealized model, to the observed variability in the Pacific storm-track entrance region, and the potential relevance to seasonal time scale variations and the Atlantic storm track.

There is considerable evidence that storm tracks are regions where upper-level wave packets coming from upstream amplify as they propagate by inducing a corresponding surface perturbation through baroclinic interactions (e.g., Wallace et al. 1988; Sanders 1988; Chang and Yu 1999; Whitaker and Barcilon 1992). Furthermore, Chang (2001) showed that, in the Pacific storm track entrance the main balance is between baroclinic growth and downstream propagation, with damping playing a relatively minor role since the eddies are still too small for barotropic shearing and other nonlinear processes to be important. Examination of daily PV maps suggests that the PV contours tend to undergo reversible sinusoidal oscillations near the Pacific stormtrack entrance region, suggesting that waves behave relatively linearly over there. Our model, therefore, is relevant to the storm-track entrance region. The degree and way in which the strength of perturbations in the storm-track entrance region affects the rest of the storm track is a separate issue, which is beyond the scope of this study. A related basic underlying assumption we made is that the variability in jet structure is externally driven. This is consistent with the observation of Chang (2001) that eddies contribute to the jet structure mostly in the Pacific exit region.

One of the main simplifications we made is that the basic state is constant in the zonal direction. This greatly reduced the needed computation time, allowing us to test many possible mechanisms and verify that the results are not sensitive to the choice of parameters. Theoretically, this should be a reasonable assumption for cases in which the zonal wavenumber is smaller than the zonal scale on which the basic state varies, and we expect it to apply at least qualitatively to the real storm tracks, but the results need to be tested in a full 3D model with a zonally varying basic state. There have been a few studies of the effects of the basic-state shear on storm-track eddy structure and growth in nonzonal basic states (e.g., Niehaus 1980, 1981; Frederiksen 1983; Pierrehumbert 1984; Cai and Mak 1990), but these studies do not address the question of the observed variability; hence it is hard to draw any relevant conclusions from them.

Another basic assumption is that the jet acts as a waveguide and the eddies are meridionally confined by the jet, with the eddies being constrained to be narrower when the jet is strong. As discussed in section 3, observed $700-\mathrm{hPa}$ heat flux does show weak negative correlation between its width and the jet strength. A caveat is that similar relations are not observed for bandpassfiltered meridional velocity (or geopotential height) variance. Currently, we do not completely understand this discrepancy between the heat flux and velocity variance. Our speculation is that during the strong jet years, since the spatial growth rate is small, the structure of the eddies are more strongly influenced by that of their upstream seed (as in our model results discussed in section 4); whereas during the weak jet years, since spatial growth is faster, the structure of the eddies more quickly takes on the normal-mode structure. Since the Pacific storm track is seeded by a northern and a southern branch (e.g., Chang and Yu 1999; Hoskins and Hodges 2002), it is conceivable that the average storm track width (in terms of velocity variance) may be broader during the strong jet years because the storm track eddies reflect more the influence from the two meridionally separated branches. On the other hand, the heat flux is a measure of the local baroclinic eddy source and is expected to reflect the modal structure more closely. Whether this is the case is an issue to be examined further using a model that incorporates zonal variations in the basic state.

As we point out in the introduction, our study seeks to explain the observed variability on interannual time scales. The negative correlation between storm track and jet strength, however, is also very prominent in the seasonal cycle (Nakamura 1992). From observations, we find that stronger jets are narrower when looking at the interannual variations of all winter months (monthly means of November-March) and also when looking at the climatological seasonal cycle (the jet is narrowest and strongest during January-February and widest and weakest during November). The change in width, however, is smaller in the seasonal cycle relative to interannual time scales. To check if this narrowing can explain the climatological seasonal cycle, we investigate the seasonal change in the spatial growth rate, by conducting stability studies based on monthly mean jet profiles from September through May, using the 3D model described in section 3 . The results, which are displayed in Table $2,{ }^{8}$ show that toward midwinter, both the temporal growth rate and group velocity increase and are maximum during January, with the growth rate effect winning over the group velocity, so that the spatial growth rate is maximum during midwinter. While the jet is slightly narrower during January than during fall or spring, the seasonal change in jet width is much smaller than the interannual change in midwinter, hence

\footnotetext{
${ }^{8}$ Here, we only show results corresponding to the medium friction case (damping timescale of 1 day), but results for the other cases are similar.
} 
TABLE 2. The growth rate, group velocity, and implied spatial growth rate of the most unstable linear modes, for the $3 \mathrm{D}$ primitive equation model runs, using basic states of various months and medium friction (see text). The most unstable zonal wavenumber for all months is 7 .

\begin{tabular}{cccc}
\hline \hline Month & Growth rate & Group velocity & $\begin{array}{c}\text { Implied spatial } \\
\text { growth rate }\end{array}$ \\
\hline Sep & 0.05 & 8.1 & 0.07 \\
Oct & 0.15 & 10.2 & 0.17 \\
Nov & 0.25 & 12.2 & 0.24 \\
Dec & 0.31 & 13.8 & 0.26 \\
Jan & 0.33 & 14.5 & 0.26 \\
Feb & 0.28 & 13.2 & 0.25 \\
Mar & 0.24 & 12.0 & 0.23 \\
Apr & 0.14 & 10.7 & 0.15 \\
May & 0.04 & 9.7 & 0.05 \\
\hline
\end{tabular}

the smaller effect on wave growth. This is consistent with the results of Chang (2001), that seasonal changes in the effects of diabatic heating (which are not incorporated in our study) are important in understanding the midwinter suppression. Note, however, that while the jet narrowing cannot account for the midwinter suppression in the climatology, it probably contributes to it during strong years (since the narrowing effects on interannual variability are much stronger in midwinter compared to fall and spring). This is consistent with the finding of Nakamura et al. (2002) that the midwinter suppression is much stronger during years when the midwinter jet is strong (and narrow).

The observed transition from a positive to negative correlation of the Northern Hemisphere midwinter storm track strength with shear occurs at jets of around $45 \mathrm{~m}$ $\mathrm{s}^{-1}$, which is roughly the upper limit for jet strength in the Atlantic (Nakamura 1992). Since our model also makes a transition from a positive to a negative correlation at roughly the same jet strengths, it is possible that the Atlantic storm track variations are governed by the same dynamics as in the Pacific, but the two happen to fall in these two separate regimes. An analysis of the jet strength versus width in the Atlantic entrance shows that stronger jets are narrower, suggesting at least that this is possible. Given, however, that the waves in the Atlantic are much more nonlinear than in the western Pacific (e.g., in terms of the ratio between eddy and mean flow quantities), the linear arguments of this study have to be applied with caution.

Observations show the Atlantic jet has a larger barotropic component, and it varies much more in latitude compared to the Pacific jet. Lee and Kim (2003) suggested these differences are a result of the Atlantic jet being eddy driven, while the Pacific jet is driven by tropical heating and it organizes the eddies. While this suggests that the two storm tracks are in different dynamical regimes, Lee and Kim (2003) also showed in an idealized model that the system makes a transition from an eddy-driven regime to a subtropical jet regime when the tropical forcing is increased. It is therefore possible that the Atlantic storm track will behave like that in the Pacific if the tropical heating, and correspondingly the jet there, ever got stronger. Our study suggests that such a change will entail changes in the interannual variations of the storm track. On the other hand, the observation that waves in the Atlantic storm track are much more nonlinear in nature, compared to waves over the Pacific entrance region, might be due to a different reason, possibly that the Atlantic storm track is seeded by already large waves from the Pacific. In this case, our linear model will probably not be relevant at all.

\section{Conclusions}

In this work, we have shown that the observed interannual variations of the Pacific storm track in its entrance region are not inconsistent with linear baroclinic instability theory, as long as the meridional structure of the jet is taken into account. Noting that the midwinter Pacific jet stream is narrower during years when it is strong, we examine the effects of jet width on the variations of wave growth with shear, using a simple quasigeostrophic channel model of downstream baroclinic growth as well as a $3 \mathrm{D}$ primitive equation model with a zonal-mean basic state. The former is done by assuming that the jet sets the meridional width of the waves. The resulting model is able to reproduce the observed weakening of the storm track with jet strength. Moreover, our model yields a negative correlation for jets stronger than some value and a positive correlation for jets weaker than that. Nakamura (1992) found a negative correlation for jets stronger than $45 \mathrm{~m} \mathrm{~s}^{-1}$ and a positive correlation below. The narrowing of the jet inhibits the local growth rate by increasing the meridional wavenumber of the perturbations. At the same time, the strengthening of the jet tends to increase the growth rate. The narrowing is most effective for large wavenumbers, resulting in the two correlation regimes. Without the narrowing of the jet, the effect of the increase in group velocity with jet strength is not sufficient to overcome that due to the increase in local growth rate. Therefore, an increase in group velocity, which has been suggested as a possible cause for the weakening during strong jet times (e.g., Nakamura 1992), cannot by itself explain the observations.

The narrowing of the jet as it strengthens can explain the decrease in storm track strength, but not the observed increase of $C_{g} /$ shear with jet strength, or the more upper-level structure for stronger jets. These observations can be explained, however, if we have an Eadylike basic state in which short waves are neutral or almost neutral. The increase in meridional wavenumber as the jet narrows causes the short-wave cutoff to move to longer zonal waves, rendering the model stable to the wavepacket perturbations coming in from upstream (assuming the zonal wavenumber of the wavepackets does not change much with shear).

Understanding the relation between the storm tracks 
and the basic state is central to understanding storm track variability. Besides the obvious effects on local weather, there is evidence that the storm tracks are important for understanding the low-frequency variability of the atmosphere, as well as for the assessment of impact of possible climate changes (see, e.g., Robinson 2000; Peng and Whitaker 1999; Held et al. 1989; Branstator 1992, 1995) because the storm tracks transport significant amounts of heat and momentum that act as forcings to the large-scale flow. Various observational studies suggest that the storm tracks have undergone major changes on decadal time scales (see Harnik and Chang 2003, and references therein). On longer timescales, storm tracks are a major source of precipitation in midlatitudes, which can be important in the growth of ice sheets during times when the ice extends to low enough latitudes. Thus, understanding storm track variability may be an important step in achieving understanding of climate variability.

Acknowledgments. Nili Harnik is supported by National Oceanic and Atmospheric Administration Grant UCSIO-CU-02165401-SCF and by a Lamont-Doherty Earth Observatory postdoctoral fellowship. Edmond K. M. Chang is supported by National Science Foundation (NSF) Grant ATM-0296076 at the State University of New York at Stony Brook. Some of the work on this paper was conducted when the authors were at the Florida State University, where they were supported by NSF Grant ATM-0003136. Nili Harnik would also like to acknowledge hospitality provided by Professor R. Lindzen at MIT. We thank Pablo Zurita-Gotor and Sukyoung Lee for very helpful comments.

\section{APPENDIX}

\section{The Effects of Surface Damping}

In this section we examine whether the addition of linear damping at or near the surface affects our results. Surface damping of momentum and temperature are the main sinks of wave activity in the storm-track entrance regions (see Chang 2001). Other damping processes, mainly upper-tropospheric nonlinear momentum damping via wave breaking, are significant more downstream. We use various combinations of Newtonian damping on temperature and Rayleigh damping on momentum, both imposed on the lowest levels of the troposphere, as well as Ekman damping. Running our model with the various forms of damping and various basic states, we find that damping does not qualitatively change the main result and that, if we take into account that the jet narrows as it strengthens, the growth rate increases, then decreases, with shear. Without taking the change in jet width into account, we find that damping leads to an increase in $S_{\mathrm{gr}}$ with shear, as Eq. (4) implies. Clearly this effect is overcome by the effects of the narrowing of the jet. Surface damping does, however, affect our results in several ways, which we present using the runs with Ekman damping.

Figure A1a shows the normal-mode growth rates for the six runs of the Charney model (basic states of Fig. 5), with a 1-day Ekman damping. We see that damping is much more effective on short waves. ${ }^{\mathrm{A} 1}$ Besides an overall reduction of growth rates, this results in an effective "shortwave cutoff." Surface damping also affects the vertical structure of the waves since it reduces wave amplitude mostly at the surface. The corresponding most unstable normal modes (not shown) are upper level, while the short-wave normal modes have a maximum at the tropopause and the surface with the latter being slightly larger, but the tropopause-to-surface amplitude ratio is larger than for the undamped runs. Based on the vertical structures, it is clear that run 6 is entirely in the short-wave regime. The "short-wave cutoff," determined from looking at the growth rates and vertical structures, shifts slightly to longer zonal wavenumbers as shear is increased in runs $1-5$. The transition to run 6 for which all zonal wavenumbers are "short" occurs quite abruptly. ${ }^{\mathrm{A} 2}$

In the previous section we argue that the modified Eady basic state is important for explaining the observed type of variability with shear, namely that waves are more upper level for strong shears, and their group propagation increases faster than linearly with shear. It seems possible that adding surface damping to the Charney model can yield similar behavior. We therefore examine the time evolution of an initial upper-level wave packet in the presence of damping. Figure A1c shows the vertical structure of wave packet envelope at its center (normalized to unit amplitude at the surface), averaged over the first week of integration, for each of the basic states (see comment in fn A2). We see that for runs 15 , the wave packets are more upper level for weak shears, but in run 6 the wave packet becomes more upper level than runs 3-5, suggestive of the observed behavior. At the same time, the group velocity increases slower than linear with shear (Fig. A2a) for runs 1-5, but it increases faster than shear between runs 5-6.

Although adding surface damping to the Charney model makes its behavior closer to that observed, the Eady-type basic state still seems more likely to explain the observed behavior. The corresponding damped runs

\footnotetext{
A1 This explains differences between our results and Farrell (1985), who found that waves in the Charney model are stabilized by Ekman damping. Farrell used a meridional wavelength of around $28^{\circ}$ latitude for a relatively weak jet ( $30 \mathrm{~m} \mathrm{~s}^{-1}$ at the tropopause) compared to our runs (wavelength of around $38^{\circ}$ latitude for a jet of $66 \mathrm{~m} \mathrm{~s}^{-1}$ ).

A2 When we add damping, the numerically obtained short-wave normal modes (all wavenumbers of run 6 fall under this category), are sensitive to the vertical resolution we use. This is due to the inability of the numerics to resolve the singularity that appears at the critical surface, when the growth rate of the mode vanishes due to the damping (see Farrell 1989). The vertical structure and growth rate estimated from the initial stage do converge, however, suggesting that the spuriously unstable short and shallow modes do not contribute significantly to the initial stages of evolution.
} 
(a) Charney w/Ekman damp.

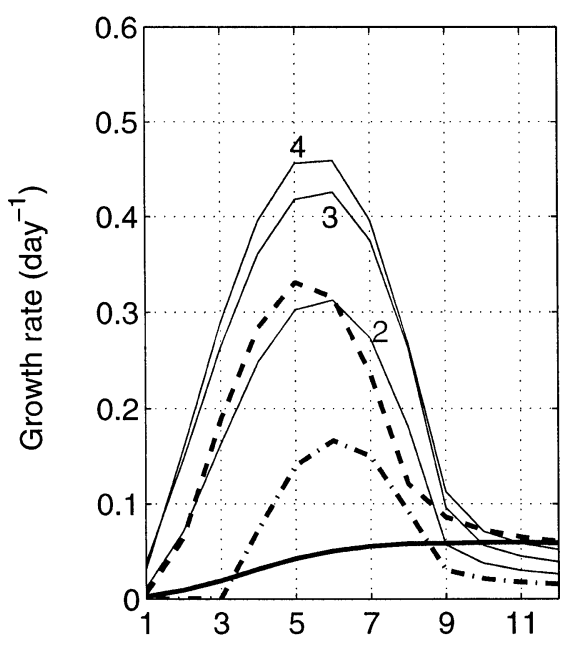

zonal wavenumber

(c) Charney, days 1-7 average

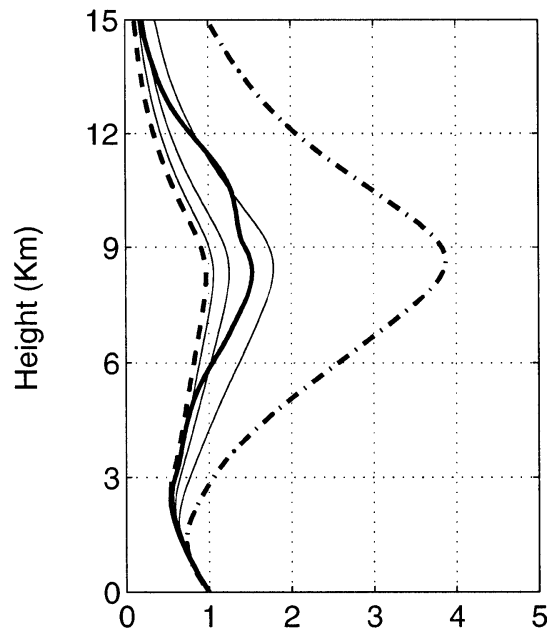

Wavepacket amplitude (b) Eady w/Ekman damp.



(d) Eady, days 1-7 average

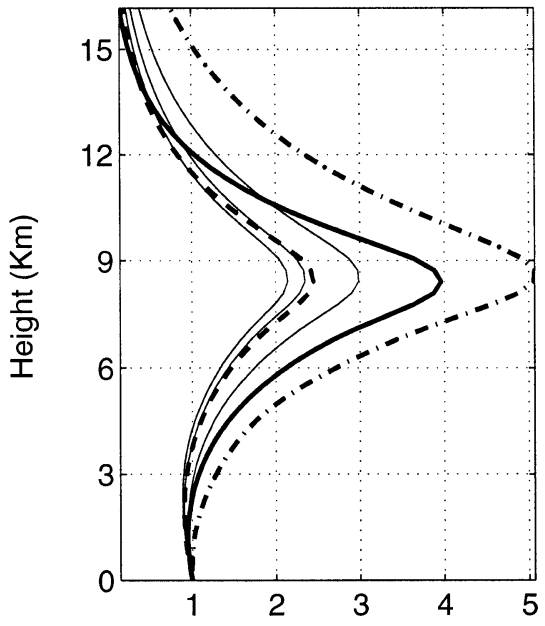

Wavepacket amplitude

FIG. A1. (a), (b) The normal-mode growth rates $\left(\right.$ day $\left.^{-1}\right)$ for the Ekman damping runs of the Charney and Eady models, respectively. (c), (d) The corresponding vertical structures of the wave packet envelopes at wave packet center, averaged over the first 7 days of the time-dependent model integrations. Thick dashed-dotted, dashed, and solid lines are for runs 1, 5, and 6, respectively.

for the modified Eady model (shown in Figs. A1b,d and Fig. A2b) show a more robust behavior, with the shifting of the short-wave cutoff to longer zonal wavenumbers being much more gradual, the ratio of tropopause to surface amplitudes increasing with shear between runs 4-6, and the group velocity increasing faster than linearly with shear between runs 4-6 (using the 0-7-day estimates, thick dashed line). In the Charney model, the short-wave normal modes are essentially tropospheresurface waves with a reduced surface amplitude while in the modified Eady model the normal modes are tropopause-surface waves. We note that the model with $\bar{q}_{y}=\beta$ and surface damping (not shown) behaves similarly to the modified Eady model because its modes are tropopause-surface waves.

Comparing the growth rates for the damped and undamped modified Eady model (Figs. A1b and 9c), we see that the effect of damping is relatively uniform on the growth rate of the unstable waves, but it slightly destabilizes the short waves [see Snyder and Lindzen 
(a) Charney, damped

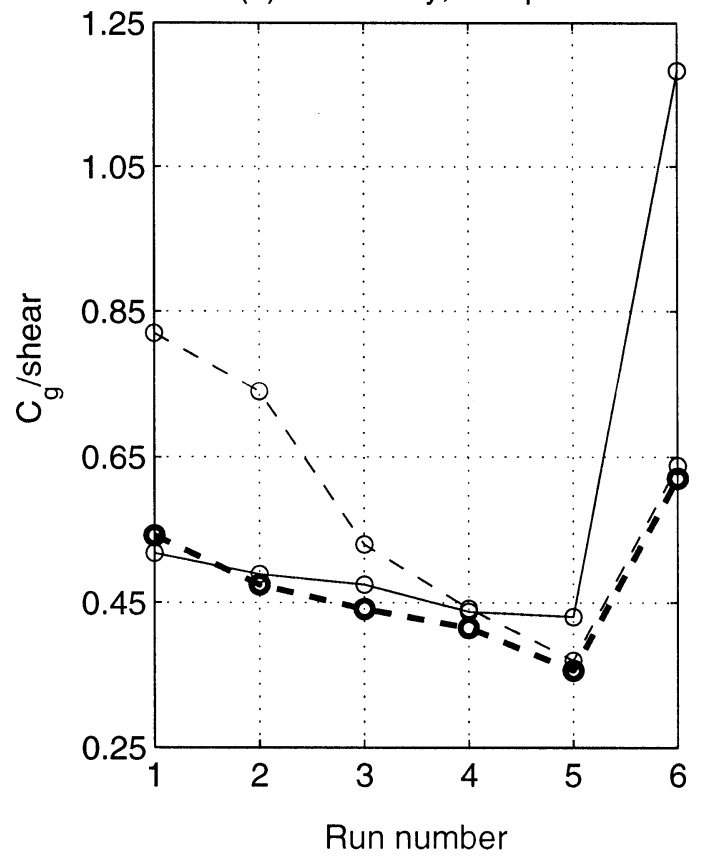

(b) Eady, damped

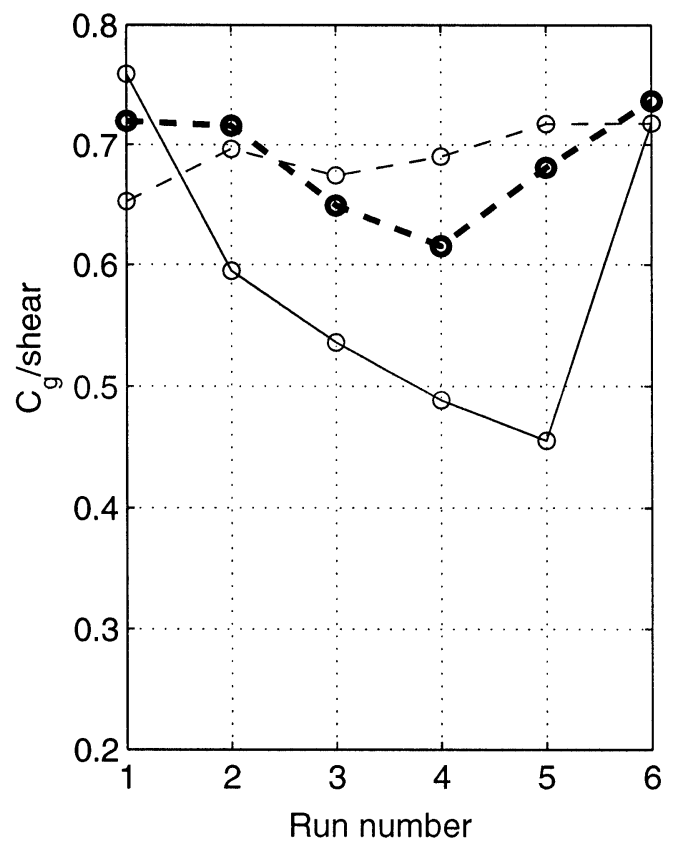

FIG. A2. As in Fig. 8 but for the corresponding damped model runs.

(1988) for an explanation]. On the other hand, damping delays the time at which the wave packets reach the normal-mode stage because the normal modes, which have a surface component, grow slower. This is evident from comparing Fig. 8 to Fig. A2, which shows that the week-based estimates of the group velocity are closer to the initial stage estimates in the damped compared to the undamped models. The overall effect is to make the transition to the almost-stable short-wave regime, as the jet is strengthened and narrowed, more gradual than in the inviscid case.

\section{REFERENCES}

Branstator, G., 1992: The maintenance of low-frequency atmospheric anomalies. J. Atmos. Sci., 49, 1924-1945.

- 1995: Organization of storm track anomalies by recurring lowfrequency circulation anomalies. J. Atmos. Sci., 52, 207-226.

Cai, M., and M. K. Mak, 1990: On the basic dynamics of regional cyclogenesis. J. Atmos. Sci., 47, 1417-1442.

Chang, E. K. M., 2001: GCM and observational diagnoses of the seasonal and interannual variations of the Pacific storm track during the cool season. J. Atmos. Sci., 58, 1784-1800.

_ and D. B. Yu, 1999: Characteristics of wave packets in the upper troposphere. Part I: Northern Hemisphere winter. J. Atmos. Sci., 56, 1708-1728.

Charney, J. G., 1947: The dynamics of long waves in a baroclinic westerly current. J. Meteor., 4, 135-163.

Eady, E. T., 1949: Long waves and cyclone waves. Tellus, 1, 33-52.

Farrell, B. F., 1982: The initial growth of disturbances in a baroclinic flow. J. Atmos. Sci., 39, 1663-1686.

, 1985: Transient growth of damped baroclinic waves. J. Atmos. Sci., 42, 2718-2727.

, 1989: Unstable baroclinic modes damped by ekman dissipation. J. Atmos. Sci., 46, 397-401.
Frederiksen, J. S., 1983: Disturbances and eddy fluxes in Northern Hemisphere flows: Instability of three-dimensional January and July flows. J. Atmos. Sci., 40, 836-855.

Harnik, N., and R. S. Lindzen, 1998: The effect of basic-state potential vorticity gradients on the growth of baroclinic waves and the height of the tropopause. J. Atmos. Sci., 55, 344-360.

—_, and E. K. M. Chang, 2003: Storm track variations as seen in radiosonde observations and reanalysis data. J. Climate, 16, $480-495$.

Held, I. M., and M. J. Suarez, 1994: A proposal for the intercomparison of the dynamical cores of atmospheric general circulation models. Bull. Amer. Meteor. Soc., 75, 1825-1830.

— - S. W. Lyons, and S. Nigam, 1989: Transients and the extratropical response to El Niño. J. Atmos. Sci., 46, 163-174.

Hoskins, B. J., and K. I. Hodges, 2002: New perspectives on the Northern Hemisphere winter storm tracks. J. Atmos. Sci., 59, 1041-1061.

— - M. E. Mcintyre, and A. W. Robertson, 1985: On the use and significance of isentropic potential vorticity maps. Quart. J. Roy. Meteor. Soc., 111, 877-946.

Ioannou, P., and R. S. Lindzen, 1986: Baroclinic instability in the presence of barotropic jets. J. Atmos. Sci., 43, 2999-3014.

Kirk-Davidoff, D. B., and R. S. Lindzen, 2000: An energy balance model based on potential vorticity homogenization. J. Climate, 13, 431-448.

Lee, S. Y., and J. L. Anderson, 1996: A simulation of atmospheric storm tracks with a forced barotropic model. J. Atmos. Sci., 53, 2113-2128.

—_, and H. K. Kim, 2003: The dynamical relationship between subtropical and eddydriven jets. J. Atmos. Sci., 60, 1490-1503.

Lindzen, R. S., 1994: The eady problem for a basic state with zero PV gradient but $\beta \neq 0$. J. Atmos. Sci., 51, 3221-3226.

Nakamura, H., 1992: Midwinter suppression of baroclinic wave activity in the Pacific. J. Atmos. Sci., 49, 1629-1642.

— , and T. Sampe, 2002: Trapping of synoptic-scale disturbances into the North Pacific subtropical jet core in midwinter. Geophys. Res. Lett., 29, 1761, doi:10.1029/2002GL015535.

, T. Izumi, and T. Sampe, 2002: Interannual and decadal mod- 
ulations recently observed in the Pacific storm track activity and east asian winter monsoon. J. Climate, 15, 1855-1874.

Niehaus, M. C. W., 1980: Instability of non-zonal baroclinic flows. J. Atmos. Sci., 37, 1447-1463.

__ 1981: Instability of non-zonal baroclinic flows: Multiple-scale analysis. J. Atmos. Sci., 38, 974-987.

Peng, S. L., and J. S. Whitaker, 1999: Mechanisms determining the atmospheric response to midlatitude SST anomalies. J. Climate, 12, 1393-1408.

Pierrehumbert, R. T., 1984: Local and global baroclinic instability of zonally varying flow. J. Atmos. Sci., 41, 2141-2162.

Rivest, C., and B. F. Farrell, 1992: Upper-tropospheric synoptic-scale waves. Part 2: Maintenance and excitation of quasi modes. $J$. Atmos. Sci., 49, 2120-2138.

— C. C. A. Davis, and B. F. Farrell, 1992: Upper-tropospheric synoptic-scale waves. Part 1: Maintenance as eady normal modes. J. Atmos. Sci., 49, 2108-2119.

Robinson, W. A., 2000: Review of WETS-The workshop on extratropical SST anomalies. Bull. Amer. Meteor. Soc., 81, 567-577.

Sanders, F., 1988: Life-history of mobile troughs in the upper westerlies. Mon. Wea. Rev., 116, 2629-2648.
Snyder, C. M., and R. S. Lindzen, 1988: Upper-level baroclinic instability. J. Atmos. Sci., 45, 2445-2459.

Swanson, K. L., P. J. Kushner, and I. M. Held, 1997: Dynamics of barotropic storm tracks. J. Atmos. Sci., 54, 791-810.

Valdes, P. J., and B. J. Hoskins, 1988: Baroclinic instability of the zonally averaged flow with boundary-layer damping. J. Atmos. Sci., 45, 1584-1593.

Wallace, J. M., G. H. Lim, and M. L. Blackmon, 1988: Relationship between cyclone tracks, anticyclone tracks, and baroclinic wave guides. J. Atmos. Sci., 45, 439-42.

Whitaker, J. S., and A. Barcilon, 1992: Type B cyclogenesis in a zonally varying flow. J. Atmos. Sci., 49, 1877-1892.

Yin, J. H., 2002: The peculiar behavior of baroclinic waves during the midwinter suppression of the Pacific storm track. Ph.D. thesis, University of Washington, $121 \mathrm{pp}$.

Zhang, Y. Q., and I. M. Held, 1999: A linear stochastic model of a GCM's midlatitude storm tracks. J. Atmos. Sci., 56, 3416-3435.

Zurita, P., and R. S. Lindzen, 2001: The equilibration of short Charney waves: Implications for potential vorticity homogenization in the extratropical troposphere. J. Atmos. Sci., 58, 3443-3462. 
Copyright of Journal of the Atmospheric Sciences is the property of American Meteorological Society and its content may not be copied or emailed to multiple sites or posted to a listserv without the copyright holder's express written permission. However, users may print, download, or email articles for individual use. 